\title{
Tracking Dopant Diffusion Pathways inside Bulk Materials
}

\author{
Stephen J. Pennycook, ${ }^{1}$ Ryo Ishikawa, ${ }^{2}$ Andrew R. Lupini, ${ }^{2}$ Scott D. Findlay, ${ }^{3}$ Rohan Mishra ${ }^{4,2}$ and \\ Sokrates T. Pantelides. ${ }^{4,2}$ \\ ${ }^{1}$ Department of Materials Science and Engineering, University of Tennessee, Knoxville, TN, USA \\ ${ }^{2}$ Materials Science and Technology Division, Oak Ridge National Laboratory, Oak Ridge, TN, USA \\ ${ }^{3}$ School of Physics, Monash University, Victoria 3800, Australia. \\ ${ }^{4}$ Department of Physics and Astronomy, Vanderbilt University, Nashville, TN, USA
}

\begin{abstract}
Aberration correction has improved both the resolution and signal to noise ratio of Z-contrast scanning transmission electron microscope (STEM) images so that it has now become possible to take movies of atomic motion [1-3]. Figure 1a-c shows an example of a Ce atom hopping between two substitutional Al sites in AlN via an interstitial location. In addition, advances in quantitative STEM have enabled accurate measurement of specimen thickness and even the three-dimensional location of a dopant atom based on comparing experimental and simulated columnar intensities in thin crystals [4-6]. In such a way the number of unit cells in each column was obtained, as shown in Fig. 1d. In thicker crystals, however, dynamical oscillations mean that column intensity alone does not allow a unique determination of dopant atom depth. However, matching additional parameters such as the image shape and peak intensity does enable a unique determination.
\end{abstract}

We used a comparison between experimental and simulated focal series to determine the fifth and third order spherical aberrations as approximately $C_{5}=10 \mathrm{~mm}$ and $\mathrm{C}_{3}=-8.3 \mu \mathrm{m}$. The probe-forming aperture half-angle was $30 \mathrm{mrad}$, and the annular detector covered $63-409 \mathrm{mrad}$. The source size was fitted by a chi-squared test giving a best fit for an effective source size of $0.62 \AA$. An example of a fit to an undoped column is given in Fig. 2a. For the two columns containing the Ce dopants, simulations were carried out for all possible atom depths. Figure $2 \mathrm{~b}$ shows how the depth of Ce atom A can be determined to single unit cell accuracy, at a depth of 9 unit cells. Atom B is similarly located to a depth of 8 unit cells. If the specimen surface was flat, this is consistent with the geometry of the jump, since the Ce dopant in column-A has two nearest neighbor Al sites in column-B, either upward or downward in depth.

Density functional theory can now be used to extract the energetics of the motion. We find vacancy mediated diffusion with an activation barrier of $0.6 \mathrm{eV}$ to be the most favorable mechanism, which explains the experimental observation of $\mathrm{Ce}$ atom frequently moving between substitutional sites. However, direct momentum transfer from the electron beam provides a useful experimental probe to study even metastable diffusion pathways in bulk solids, such as, in this case, diffusion through an interstitial site. If the $\mathrm{Ce}$ atom jump to the interstitial site, which has an activation barrier of $3.1 \mathrm{eV}$, were thermally activated we would need to wait $10^{33}$ years at room temperature, or heat the sample to $1300 \mathrm{~K}$, to observe a single jump per second.

It is interesting to speculate what improved sensitivity through aberration correction to higher angles might bring. A $60 \mathrm{mrad}$ aberration corrected probe would have a free space depth of focus below $1 \mathrm{~nm}$, and with the enhanced peak intensity and ensuing signal to noise ratio improvement, it would greatly enhance our ability to study point defect configurations in three dimension inside crystals, along with their metastable configurations and migration dynamics. 


\section{References}

[1] J. Lee et al., Nature Communications, 4, 1650 (2013).

[2] W. Zhou et al., Phys Rev Lett 109, 206803 (2012).

[3] T. Pennycook et al., Nano Lett 12, 3038 (2012).

[4] J.M. LeBeau et al., Nano Lett. 10, 4405 (2010).

[5] R. Ishikawa et al., Microsc. Microanal. 20, 99 (2014).

[6] J.Hwang, et al., Phys Rev Lett 111, 266101 (2013).

[7] Research sponsored by a JSPS postdoctoral fellowship for research abroad (RI), the Materials Sciences and Engineering Division, Office of Basic Energy Sciences, U.S. Department of Energy (SJP, RM, STP) and by the McMinn Endowment (STP) at Vanderbilt University. Computations were supported by the National Energy Research Scientific Computing Center.

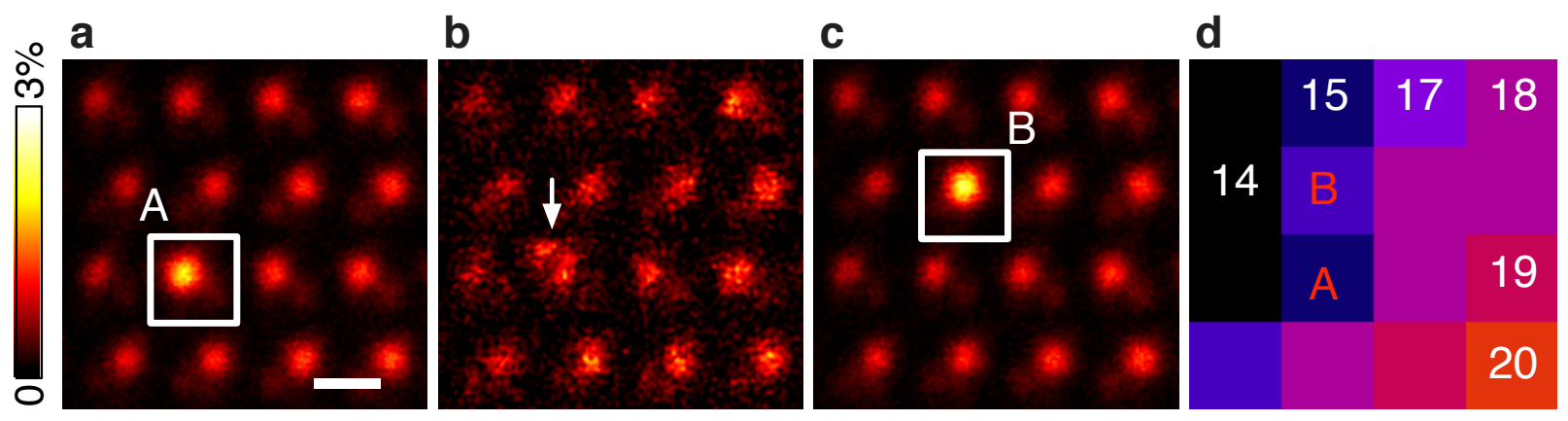

Fig. 1: Z-contrast images extracted from a time sequence of images of $w$-AIN viewed along the [1120] direction, (a) average image over frames 1-19, (b) frame 20, (c) average image over frames 21-40. The Ce dopant atom jumps from site A to B through an interstitial location. (d) Thickness map showing the number of atoms per column.
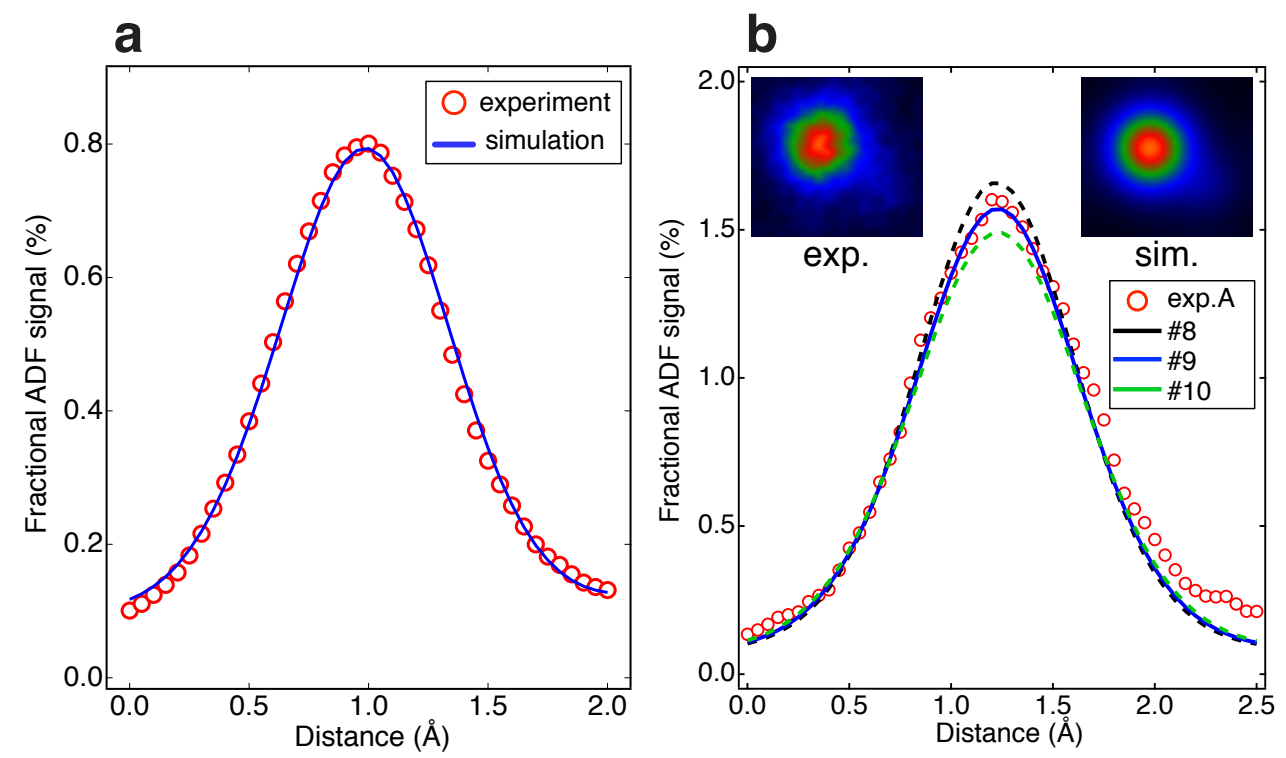

Fig. 2: Fit of (a) undoped columnar profile to frozen phonon image simulations, (b) fit to doped column A showing the Ce to be located 9 unit cells deep. 